\title{
On the Selection of Optimum QoS Bounds for Mobile IP Enabled VANET through Realistic City Scenario
}

\author{
Kanu Priya ${ }^{1 *}$ and Jyoteesh Malhotra ${ }^{2}$ \\ 'Department of Computer Science and Engineering, GNDU Regional Campus, Jalandhar, India; \\ kanupriya2310@gmail.com \\ 2Department of Electronics and Communication Engineering, GNDU Regional Campus, Jalandhar, India; \\ jyoteesh@gmail.com
}

\begin{abstract}
The emergence of DSRC 802.11p in VANET (Vehicular Adhoc Network) has resulted in the evolution of Intelligent Transport System (ITS). However, one of the major challenges in the success of VANET is the robust and reliable implementation through rapid vehicle movement and hostile channel conditions. Mobile IP can be considered as one of the most promising technologies to address the challenge of uninterrupted services in spite of rapid movement. In this work, a Mobile IP enabled scenario has been proposed and the hostile channel conditions have been modeled using Rayleigh fading distribution. The optimum QoS bounds have been obtained through extensive simulations by varying the queue size and application traffic type. Due to the quick handoff in Mobile IP, there is crucial need of selection of appropriate queue size responsible for accommodating data packets. Considering all these factors, this paper emphasizes on the selection of most suitable application traffic along with appropriate queue size even under realistic channel conditions for Mobile IP enabled City Scenario.
\end{abstract}

Keywords: ITS, Mobile IP, PCR, PDR, Rayleigh Fading

\section{Introduction}

Lately a number of communication technologies have been invented but the development of DSRC $802.11 \mathrm{p}$ by successful allotment of $5.9 \mathrm{GHz}$ frequency band has become a boon for VANET. Vehicular Adhoc Networks are also anticipated in the deployment of Mobile IP for accommodating host mobility by the enhancement of current Internet Protocol (IP) [1]. In the literature it has reviewed that more stress is laid on the routing overheads and handoff management. Handoff generally occurs when a mobile node shifts its point of attachment from one access point to the other. These handoff further increases in packet loss ratio. $\mathrm{In}^{2}$ mobile IP has been deployed in two different propagation models i.e. 2 Ray Ground and Shadowing propagation model using $802.11 \mathrm{~g}$ MAC Interface at TCP traffic in NS2 and is concluded that performance of mobile IP highly depends upon the prop- agation channel model selected. The advanced FMIPV6 (Fast Mobile Internet Protocol Version-6) in ${ }^{3}$ has been compared with standard FMIPV6 using NS2 Simulator in ideal environment. Hence these techniques need to be evaluated in more realistic highway and city scenarios (by considering fading and shadowing environments) ${ }^{4}$. $\operatorname{In}^{5,6}$ also Mobile IP has been investigated in ideal conditions only. So to fill all such gap areas work has been done here under realistic channel conditions. This paper intends to find out effect of varying queue size for AWGN (No Fading) and Rayleigh Fading environment. For achieving this realistic scenario incorporated with 802.11p ITS Cars has been presented in this paper using two application traffic types namely stg/rtg and stcp/rtcp. The performance evaluations of No Fading and Rayleigh Fading have been done in terms of QoS parameters namely Packet Collision Rate, Packet Drop Rate and Throughput Rate in Mobile IP enabled VANET. 
The rest of the paper is constructed as:

Section II explains background of Mobile IP and Rayleigh fading; Section III highlights Simulation Environment used; Section IV throws light on Simulation Results obtained and its discussion and at last Section V concludes the paper.

\section{Background}

\subsection{Rayleigh Fading}

Rayleigh fading may be defined as the propagation environment when no LOS communication or there is existence of multiple indirect paths between sender and receiver. The objects present in the communication path reflect, refract, diffract and attenuate the signal as a result the received signal is totally different from the transmitted signal. The stochastic Fading model for this propagation environment is called Rayleigh distribution model $^{7}$. Rayleigh fading is also called as Small Scale

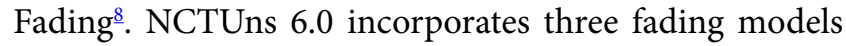
namely No Fading (AWGN), Rayleigh Fading and Ricean Fading. In this paper we have focused on AWGN and Rayleigh Fading models.

\subsection{Mobile IP}

The concept of Mobile IP in VANET facilitates not only long range communication but also help to trace the location of vehicles. Mobile IP was defined by IETF (Internet Engineering Task Force) in 2002采. In mobile IP two agents are responsible for tunneling the data packets to the mobile node (vehicle). One is the Home Agent which is a RSU in the home network and other one is foreign agent (also a RSU) that delivers packets to mobile node (vehicle) when it comes in its communication range. The Mobile IP works in three steps namely Agent Discovery, Registration and Tunneling ${ }^{10}$. Considering Mobile IP as an important aspect in VANET, the performance of AWGN and Rayleigh Fading models have been evaluated at different queue sizes using stg/rtg and stcp/rtcp application data.

\section{Simulation Environment}

This section presents the simulation scenario for computing the desired performance through realistic channel conditions for Mobile IP enabled VANET. Both network and traffic simulation capabilities are required in VANET. Considering this as an important aspect, NCTUns 6.0 a GUI based tool has been used in this research work. In this paper a scenario has been presented for evaluating the effect of increased queue size at two application traffic types namely stg (send traffic grapher); rtg (receiver traffic grapher) and stcp (sender transmission control protocol) and rtcp (receiver transmission control protocol). The hostile channel conditions have also been considered through Rayleigh Fading distribution to determine the effect of varied queue size on aforementioned traffic types. The performance evaluations in AWGN and Rayleigh Fading environment at different queue sizes (mentioned in table 1) have been done in terms of QoS parameters namely Packet Collision Rate, Packet Drop Rate and Throughput Rate in Mobile IP enabled city scenario. The simulation scenario has been constructed using 'Draw' topology feature. The scenario consists of three RSU's (having their individual subnets) labeled with id 8, 9 and 11; four agent controlled 802.11p ITS cars; a router and host for generating traffic at sender side. There are two types of traffic generation commands used i.e. stg/ rtg and stcp/rtcp in both AWGN and Rayleigh Fading. The parameters of physical layer and channel model have been set using 'Edit' topology feature of NCTUns. The parameters are shown in table 1.

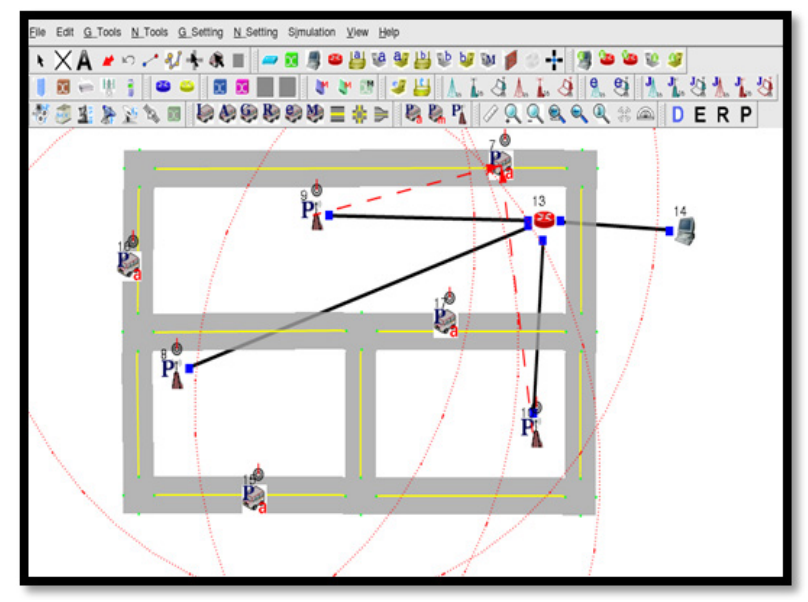

Figure 1. Simulation Scenario.

Table 1. Simulation parameters

\begin{tabular}{|l|l|}
\hline Parameters & Specification \\
\hline MAC & $802.11 \mathrm{p}$ \\
\hline Speed $(\mathrm{m} / \mathrm{sec})$ & 20 \\
\hline Application traffic & stg, rtg, stcp, rtcp \\
\hline
\end{tabular}




\begin{tabular}{|l|l|}
\hline Queue Size (packets) & $10,25,50,75,100$ \\
\hline Communication & Full Duplex \\
\hline Fading & AWGN, Rayleigh Fading \\
\hline Fading Variance & 10 \\
\hline $\begin{array}{l}\text { Transmission } \\
\text { Power(dbm) }\end{array}$ & 15 \\
\hline Queue & FIFO \\
\hline Frequency (MHz) & 2400 \\
\hline $\begin{array}{l}\text { Simulation } \\
\text { time(seconds) }\end{array}$ & 400 \\
\hline
\end{tabular}

\section{Results and Discussion}

The following graphs highlight the results obtained using stg/rtg and stcp/rtcp application traffic in AWGN (No Fading) in terms of QoS parameters at different queue sizes. Secondly the performance parameters at 10 packet queue size are compared in both fading models using aforementioned traffic types. Fig 2 and Fig 3 depicts the Ideal Packet Collision Rate using stg/rtg and stcp/ rtcp application traffic at five different queue sizes i.e. $10,25,50,75,100$ packets. It is examined that with increase in queue size, the number of packet collisions also starts increasing. As the queue size increases, more packets are accommodated in a queue and as a result they collide with each other and hence greater PCR. The maximum Packet Collision Rate is achieved at 100 packets queue size in both stg/rtg and stcp/rtcp with an average value of 4.99 and 5.88 respectively. It is also evaluated that number of collisions in stcp/rtcp are more than stg/rtg application traffic at all the queue sizes. Fig4 and Fig5 depict the Packet Drop Rate. It is observed that as the queue size increases, PDR drops. The minimum PDR is achieved at

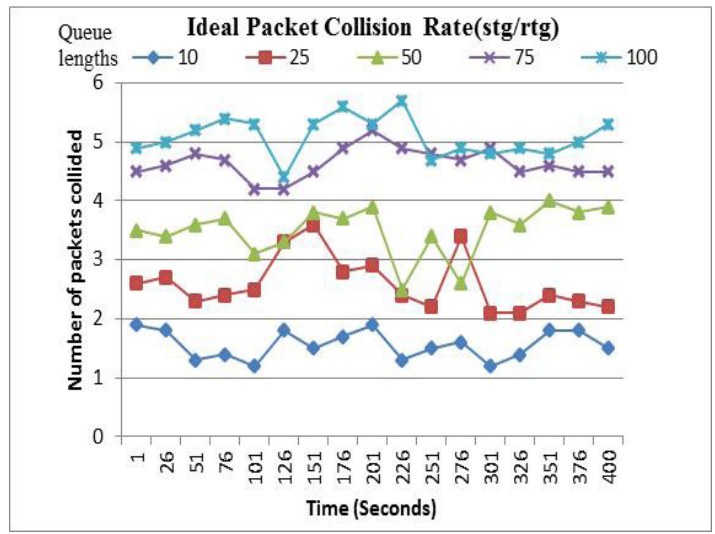

Figure 2. Ideal Packet Collision Rate using stg/rtg application. traffic
100 packets queue size in both stg/rtg and stcp/rtcp with an average value of 1.10 and 3.89 respectively. Fig6 and Fig7 highlight the Throughput Rate achieved using different application data. The maximum Throughput Rate is achieved using stg/rtg at 10 packets queue size with an average value of 19.69 whereas maximum Throughput value achieved by stcp/rtcp is lesser than $\mathrm{stg} / \mathrm{rtg}$ by $6.12 \%$.

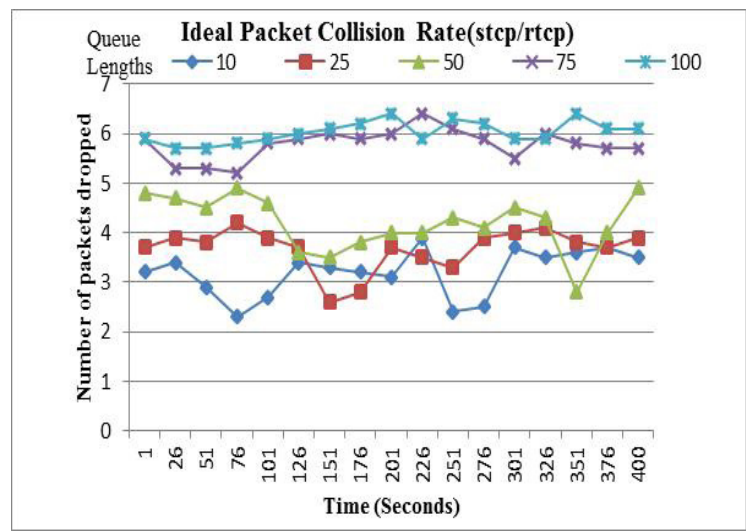

Figure 3. Ideal Packet Collision Rate using stcp/rtcp application traffic.

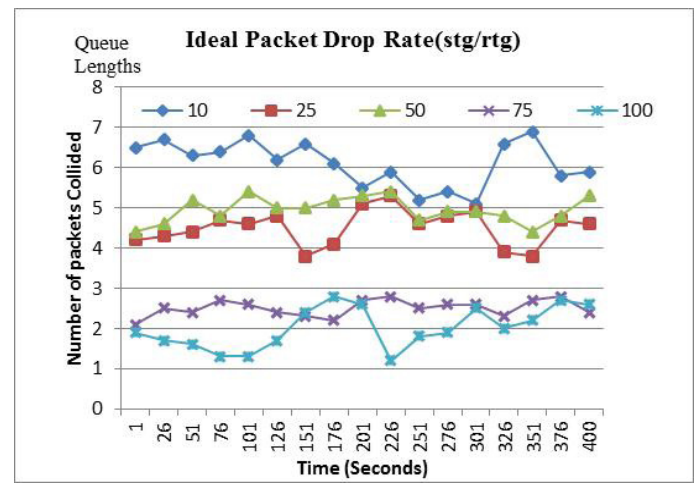

Figure 4. Ideal Packet Drop Rate using stg/rtg application traffic.

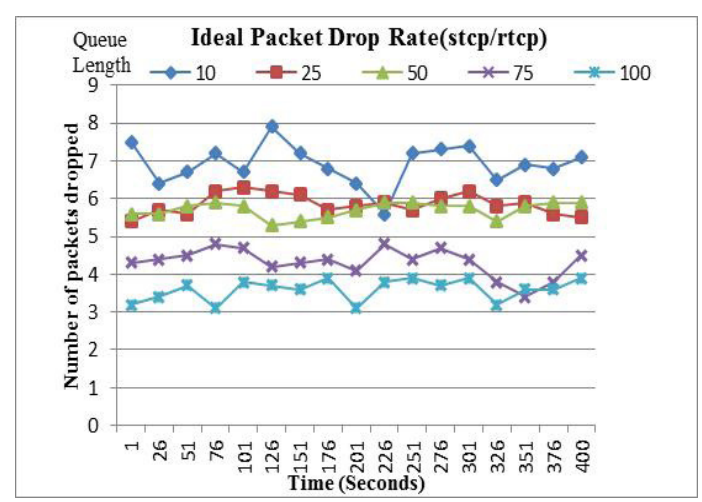

Figure 5. Ideal Packet Drop Rate using stcp/rtcp application traffic. 


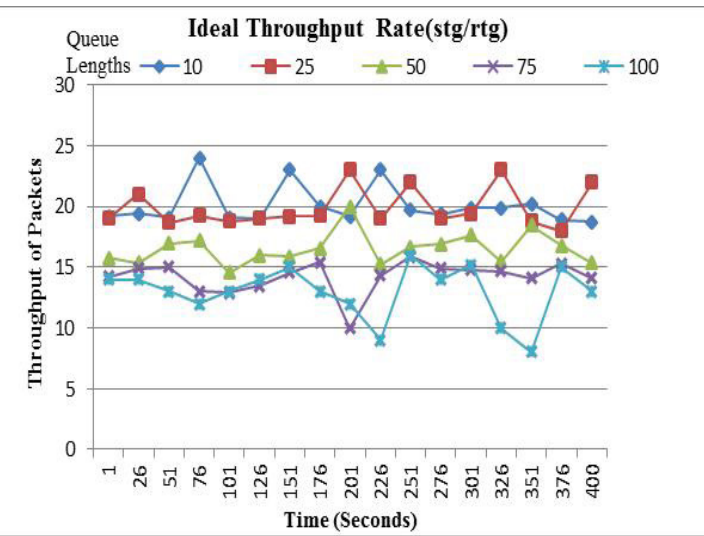

Figure 6. Ideal Throughput Rate using stg/rtg application traffic.

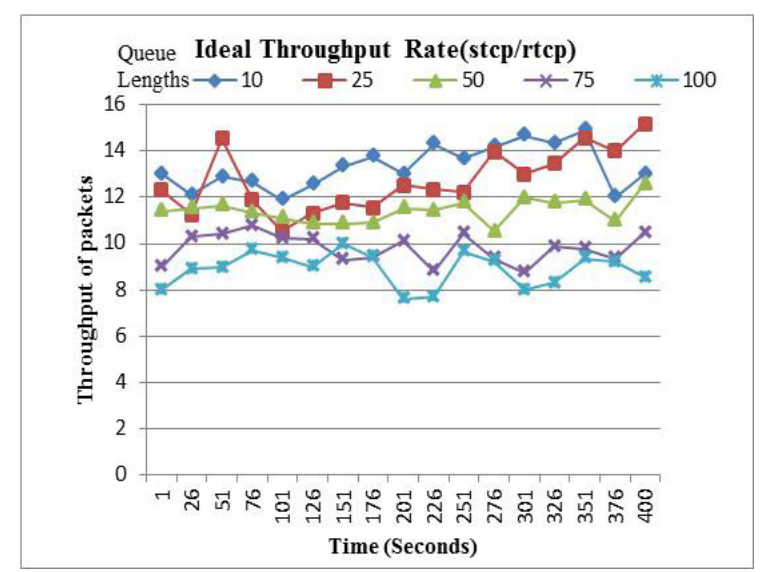

Figure 7. Ideal Throughput Rate using stcp/rtcp application traffic.

\subsection{Effect of Hostile Channel Conditions}

The following graphs show the comparative analysis of AWGN and Rayleigh Fading at 10 packets queue size in terms of QoS parameters for Mobile IP enabled city scenario. It is determined that when Rayleigh Fading is introduced, the performance of both traffic types degrade. Fig8 and Fig9 show the Packet Collision Rate. It has been observed that for stg/rtg and stcp/rtcp application data the PCR for Rayleigh Fading is more than AWGN by $4.1 \%$ and $3.63 \%$ respectively. Fig 10 and Fig 11 depict Packet Drop Rate at both application data. It has been observed that due to the incorporation of Rayleigh Fading, the number of dropped packets increase. PDR in Rayleigh Fading is exceeding AWGN for stg/rtg by $1.05 \%$ and $3.75 \%$ in stcp/rtcp. Fig 12 and Fig 13 throw light on the Throughput Rate achieved in both fading models. It is examined that the Throughput Rate is high for No Fading Model. In Rayleigh Fading, the through- put achieved is comparatively lesser for both application data. As expected, with incorporation of Rayleigh Fading model at both application traffic types, the performance of VANET deteriorates. More specific average values of all performance parameters have been tabulated in table 2 .

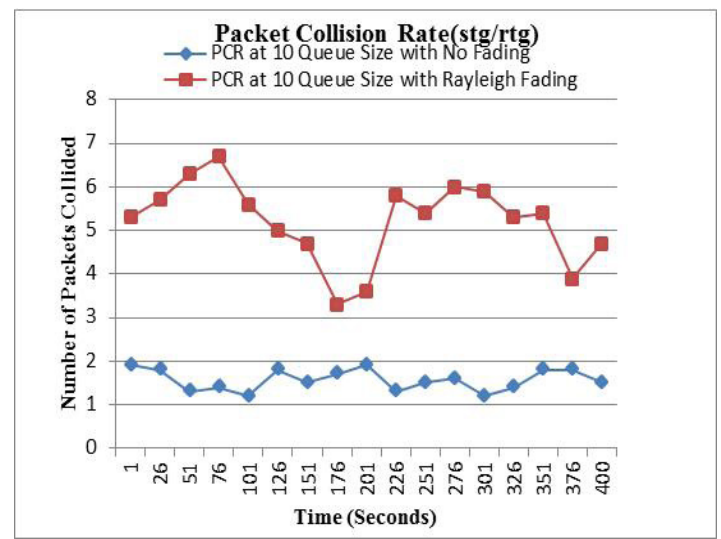

Figure 8. Packet Collision Rate using stg/rtg in both AWGN and Rayleigh Fading at 10 packets Queue Size.

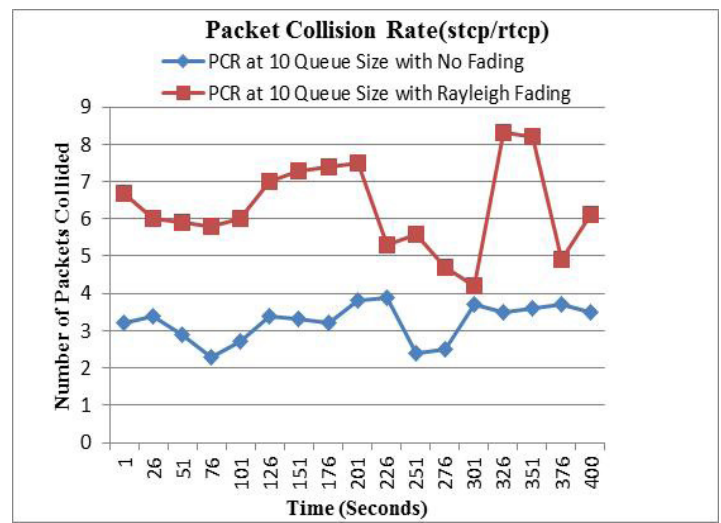

Figure 9. Packet Collision Rate using stcp/rtcp in both AWGN and Rayleigh Fading at 10 packets Queue Size.

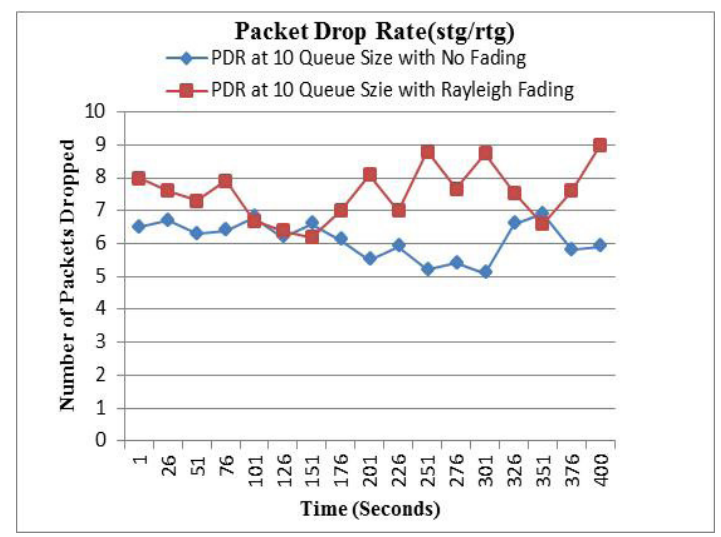

Figure 10. Packet Drop Rate using stg/rtg in both AWGN and Rayleigh Fading at 10 packets Queue Size. 


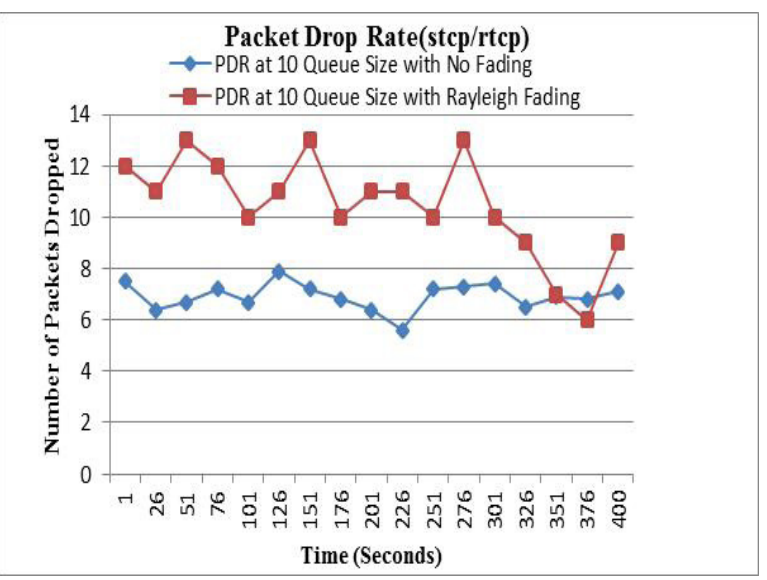

Figure 11. Packet Drop Rate using stcp/rtcp in both AWGN and Rayleigh Fading at 10 packets Queue Size.

The above table describes the average values of QoS parameters in Mobile IP at two application traffic types namely stg/rtg and stcp/rtcp at five different queue sizes in
AWGN (No Fading) and Rayleigh Fading. Through these results it has been analysed that $\mathrm{stg} / \mathrm{rtg}$ application traffic gives superior results than stcp/rtcp. It has been examined that the performance of stg/rtg gives better results in the absence of Rayleigh Fading Model. For Rayleigh Fading there is more packet loss and hence it has greater Packet Collision Rate and Packet Drop Rate. Greater packet loss results in decrease in Throughput Rate. For instance at 10 packets queue size, the Packet Collision Rate in Rayleigh Fading Model exceeds AWGN by $4.05 \%$ and with increase in queue size this value further increases. Packet Drop Rate in Rayleigh Fading Model keeps on increasing with increase in queue size whereas decreases for AWGN. The maximum PDR is achieved at 10 packets queue size in Ideal Environment with an average value of 6.99 whereas it is 12.027 for Rayleigh Fading at 100 packets queue size. Due to increase in packet collisions and packet drop rate, the Throughput Rate in Rayleigh Fading is less. Hence

Table 2. (Simulation Results in No Fading and Rayleigh Fading Models)

\begin{tabular}{|c|c|c|c|c|c|}
\hline \multirow[b]{2}{*}{$\begin{array}{c}\text { QoS } \\
\text { parameters } \\
\downarrow\end{array}$} & \multirow[b]{2}{*}{$\begin{array}{l}\begin{array}{l}\text { Application } \\
\text { traffic }\end{array} \\
\longrightarrow \\
\text { Queue } \\
\text { size(packets) } \\
\downarrow\end{array}$} & \multicolumn{2}{|l|}{ AWGN } & \multicolumn{2}{|c|}{$\begin{array}{l}\text { RAYLEIGH } \\
\text { FADING }\end{array}$} \\
\hline & & Stg/rtg & Stcp/rtcp & Stg/rtg & Stcp/rtcp \\
\hline \multirow{5}{*}{ PCR } & 10 & 1.387 & 3.590 & 5.431 & 7.221 \\
\hline & 25 & 2.392 & 3.657 & 5.441 & 8.338 \\
\hline & 50 & 3.437 & 4.701 & 7.467 & 8.431 \\
\hline & 75 & 4.476 & 5.751 & 8.487 & 9.610 \\
\hline & 100 & 4.991 & 5.889 & 9.997 & 11.042 \\
\hline \multirow{5}{*}{ PDR } & 10 & 6.995 & 7.312 & 8.045 & 11.07 \\
\hline & 25 & 4.044 & 5.449 & 9.092 & 12.08 \\
\hline & 50 & 4.069 & 5.339 & 10.099 & 12.101 \\
\hline & 75 & 2.077 & 4.546 & 10.119 & 13.394 \\
\hline & 100 & 1.106 & 3.897 & 12.027 & 14.357 \\
\hline \multirow{5}{*}{ TR } & 10 & 19.690 & 13.570 & 14.786 & 11.630 \\
\hline & 25 & 19.639 & 12.563 & 13.663 & 10.629 \\
\hline & 50 & 15.629 & 11.433 & 13.623 & 7.628 \\
\hline & 75 & 14.620 & 10.423 & 10.615 & 7.602 \\
\hline & 100 & 14.310 & 10.307 & 8.518 & 5.143 \\
\hline
\end{tabular}


it can be concluded that due to the incorporation of Rayleigh Fading, the performance of VANET worsens.

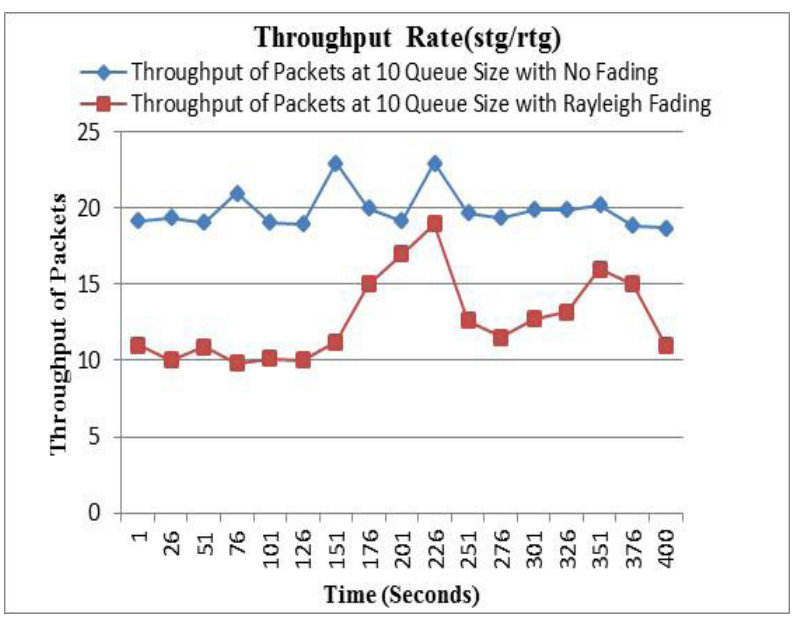

Figure 12. Throughput Rate using stg/rtg in both AWGN and Rayleigh Fading at 10 packets Queue Size.

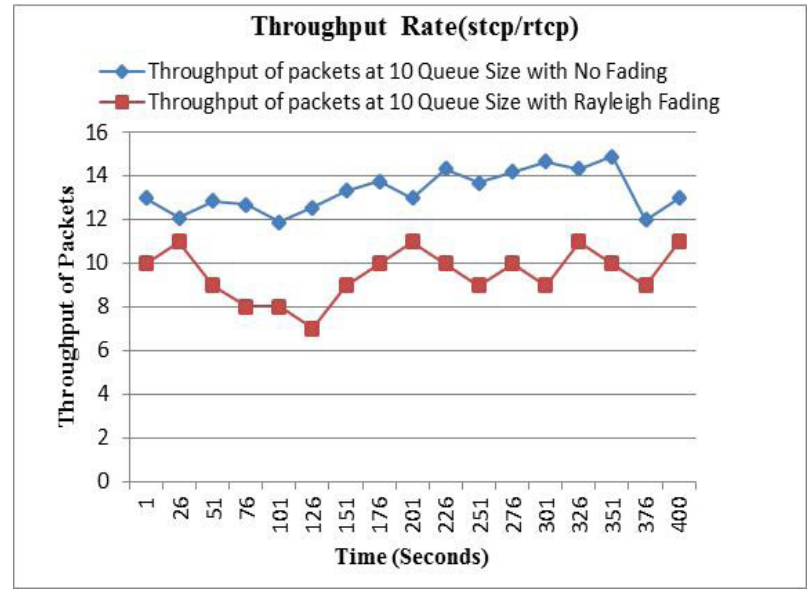

Figure 13. Throughput Rate using stcp/rtcp in both AWGN and Rayleigh Fading at 10 packets Queue Size.

\section{Conclusion}

In this paper we have analysed the impact of varying queue size at two different application traffic types in Mobile IP for Realistic City Scenario. Due to the quick handoff in Mobile IP, there is crucial need of selection of appropriate queue size responsible for accommodating data packets. Considering all these factors, this paper emphasizes on the selection of most suitable application traffic along with appropriate queue size even under realistic conditions. Through simulative investigations it has been determined that for both models the most appropriate results are achieved at smaller queue size i.e. at 10 packet queue size with less number of collisions, Packet Drop Rate and high values of Throughput Rate. Even though congestion detection and other control services are not present in stg/rtg application traffic but superior performance achieved in terms of QoS parameters determines that $\mathrm{stg} / \mathrm{rtg}$ is the best suitable application traffic. As expected, the performance of VANET deteriorates with the incorporation of Rayleigh Fading Model. Through extensive simulations carried out in this work it has been found that the optimum queue size for Mobile IP enabled realistic city scenario is of 10 packets and the most appropriate traffic type is stg/rtg.

\section{References}

1. Perkins C. IP mobility support. Technical Report RFC 2002. IETF. 1996 Oct.

2. Janevski T, Petrov I. Analysis of Mobile IP for NS-2," $16^{\text {th }}$ Telecommunication forum TELFOR 2008 Nov; 191-4.

3. Koodli R et al. FMIPv6, IETF, RFC.4068,2005.

4. Dahiya P, Deswal S. Performance Analysis of Advanced FMIPv6 for Handover Management In VANET. International Journal of Innovative Research in Science, Engineering and Technology. 2014 Jun; 3(6):13572-8,

5. Samad M, Herman SH. Quality of Service for Mobile IP Services in Wireless Network. Asia Pacific Conference on Applied Electromagnetic, Johor. 2005 Malaysia.

6. Firouzjaee SG, Fathy M, Raahemifar K. Utilizing Mobile IP, MPLS to Improve QoS in VANET. International Conference on Advances in Signal Processing and Communications (SPC- 2012) Amsterdam, Netherlands. 2012 Jun 8. p. 122-5.

7. Kumar S, Gupta PK. Performance Analysis of Rayleigh and Rician Fading Channel Models using Matlab Simulation. I.J. Intelligent Systems and Applications. 2013; 94-102.

8. Rappaport TS. Wireless Communications, Chs. 3 and 4, Upper Saddle River, NJ: Prentice Hall, 1996.

9. Wang S-Y, NCTUns 5.0: A Network Simulator for IEEE 802.11(p) and 1609 Wireless Vehicular Network Researches. 2013 Jan; 1.

10. Perkins CE. Sun Microsystems. IEEE Communications Magazine. 1997; 84-99.

11. Sommer C, Dressler F.Using the right two-ray model, A measurement based evaluation of PHY models in VANETs. Proc ACM Mobi Com. 2011; 1-3.

12. Cespedes.S, Taha S, Xuemin S. A Multihop- Authenticated Proxy Mobile IP Scheme for Asymmetric VANET. Vehicular Technology, IEEE Transactions . 2013; 62(7).

13. Bechler M, Wolf L. Mobility Management for Vehicular Ad-Hoc Networks. Vehicular Technology Conference, 2005 VTC 2005-Spring, IEEE 2005; 61st. 4:;2294-8. 
14. Al Shidhani A, Leung VCM. Secure and efficient multihop mobile IP Registration scheme for MANET-Internet inte- grated architecture. Proc IEEE Wireless Communication Networking Conference. 2010; 1 -6. 